\title{
PAPER
}

\section{A randomised controlled trial comparing rehabilitation against standard therapy in multiple sclerosis patients receiving intravenous steroid treatment}

\author{
J Craig, C A Young, M Ennis, G Baker, M Boggild
}

See end of article for authors' affiliations

Correspondence to:

Correspondence to:
Miss J Craig, Research Office, NRU, Walton

Centre for Neurology \&

Neurosurgery, Lower Lane,

Liverpool L9 7L, UK;

jenny.craig@

thewaltoncentre.nhs.uk

Received 13 May 2002

In revised form

11 November 2002

Accepted

21 November 2002
Background: There is evidence to support both the use of intravenous methylprednisolone (IVMP) in multiple sclerosis (MS) relapse and physiotherapy in the management of MS, but no studies have investigated the combination of steroids and rehabilitation together.

Objectives: To evaluate the benefits of IVMP with planned, comprehensive multidisciplinary team (MDT) care compared to IVMP with standard care.

Methods: In this randomised controlled trial, patients confirmed to have had a definite MS relapse severe enough to warrant IVMP (1 g daily for three days) were randomised to two groups. The control group was managed according to the standard ward routine; the treatment group received planned coordinated multidisciplinary team assessment and treatment. Baseline assessments, including demographics and Expanded Disability Status Scale (EDSS) were carried out on both groups. The primary outcome measures were Guy's Neurological Disability Scale (GNDS), and Amended Motor Club Assessment (AMCA). The secondary measures were the Barthel Index (BI), Human Activity Profile (HAP), and Short Form Item 36 Health Survey (SF-36). All measures have published data on reliability and validity. Measures were administered at one and three months.

Results: Forty subjects, including 27 females, completed data collection. There were no significant differences between the two groups at baseline. Results showed statistically significant differences in GNDS ( $p=0.03), \operatorname{AMCA}(p=0.03), \operatorname{HAPM}(p<0.01), \operatorname{HAPA}(p=0.02)$, and BI $(p=0.02)$ at three months in favour of planned MDT care.

Conclusion: This study indicates that combining steroids with planned MDT care is superior to administering them in a standard neurology or day ward setting. Further research is necessary in order to confirm this finding.
M ultiple sclerosis (MS) is the most common cause of progressive neurological disability in young adults in the United Kingdom. ${ }^{1}$ The course of MS varies, but four patterns have emerged, as described by Lublin and Reingold. ${ }^{2}$ These subtypes are distinguished by the time course of relapse and progression. ${ }^{3}$ Current medical management falls into three broad categories: disease modifying therapies, symptom management, and relapse management. The mainstay of relapse management is steroid therapy, usually intravenous methylprednisolone (IVMP).

The use of steroids in MS has been advocated since the 1940s and 1950s. ${ }^{4}$ Their exact mode of action is unclear, but it is thought that they act to reverse breakdown of the blood-brain barrier, ${ }^{56}$ thus accelerating recovery from the relapse. ${ }^{1}$ There is extensive literature evaluating the benefits of steroids. The Cochrane review of corticosteroids for MS relapse $^{7}$ determined the efficacy and safety of steroids in reducing the morbidity from MS. Six trials covering 377 subjects experiencing relapses irrespective of their disease course indicated that intravenous administration may be more efficacious than oral administration of methylprednisolone. ${ }^{7} \mathrm{~A}$ study on views of British neurologists on steroid administration suggested that, in the UK, delivery of IVMP varied in dose, mode, and venue for treatment. ${ }^{8}$

It is also believed that physical therapy, directed at normalising tone and improving movement and posture ${ }^{10}$ during steroid therapy and the acute period afterwards, facilitates improved longer term functional outcome. Anecdotal evidence suggests that many patients, due to the timing of their admission for IVMP, do not receive adequate rehabilitation or education regarding appropriate care after discharge.
Studies conducted on the benefits of physiotherapy (PT) and occupational therapy $(\mathrm{OT})^{11-15}$ generally have shown therapy to be of benefit to MS patients. Rehabilitation addresses many issues and should be viewed as an opportunity for gains to be made in various aspects of the person's life, encompassing both physical and emotional aspects. Recent studies ${ }^{16}{ }^{17}$ have shown that inpatient rehabilitation by a multidisciplinary team (MDT) is beneficial for people with MS and that some benefits are sustained for periods of up to six months in the community. Further studies also found that limited periods of rehabilitation helped in reducing disability and handicap in the short term. ${ }^{18}{ }^{19}$ Patients being treated for relapse should be considered for rehabilitation, but none of these studies included relapsing patients and none of the studies covered in the Cochrane review included any rehabilitation element in their design.

A study conducted recently concluded that other rehabilitative interventions should be considered alongside steroid treatment. ${ }^{20}$ The results of this study indicated that although impairment and disability did improve after steroid treatment, perceived health status only slightly improved. The authors concluded that rehabilitation intervention with steroid

Abbreviations: AMCA, Amended Motor Club Assessment; BI, Barthel Index; EDSS, Expanded Disability Status Scale; GNDS, Guy's Neurological Disability Scale; HAP, Human Activity Profile; IVMP, intravenous methylprednisolone; MDT, multidisciplinary team; MS, multiple sclerosis; OT, occupational therapy; PT, physiotherapy; SF-36, Short Form 36 Item Health Survey 
Table 1 Outcome measures scoring

\begin{tabular}{|c|c|c|c|}
\hline Measure & $\begin{array}{l}\text { No. of } \\
\text { items }\end{array}$ & Description of scoring & $\begin{array}{l}\text { Level of change score for clinical } \\
\text { significance }\end{array}$ \\
\hline Guy's Neurological Disability Scale (GNDS) & 12 & $\begin{array}{l}\text { Range }=0-60 \\
0=\text { normal status } \\
60=\text { maximum possible disability }\end{array}$ & 5 \\
\hline Amended Motor Club Assessment (AMCA) & 30 & $\begin{array}{l}\text { Range }=0-76 \text { (excluding upper limb section) } \\
0=\text { poor motor function } \\
76=\text { normal motor function }\end{array}$ & 8 \\
\hline Human Activity Profile (HAP) & 94 & $\begin{array}{l}\text { Range }=0-94 \\
0=\text { low activity levels } \\
94=\text { normal activity levels }\end{array}$ & Unavailable \\
\hline Short Form 36 Health Survey (SF-36) & 36 & $\begin{array}{l}\text { Range }=0-100 \\
0=\text { low levels of health related quality of life } \\
100=\text { high levels of health related quality of life }\end{array}$ & 10 \\
\hline Revised Barthel Index (BI) & 10 & $\begin{array}{l}\text { Range }=0-20 \\
0=\text { high dependency } \\
20=\text { independence }\end{array}$ & 2 \\
\hline
\end{tabular}

treatment may be more effective in improving subjective disease state and suggested a randomised study should address this issue.

In view of this lack of clinical and published evidence, the aim of this randomised trial was to evaluate the benefits of receiving a combination of IVMP and focused MDT management of relapse, compare to IVMP alone. The experimental hypothesis was that steroid therapy for MS patients in relapse combined with focused multidisciplinary team care was more beneficial than steroid therapy alone.

\section{METHODS}

\section{Patients and protocol}

This was conducted as a randomised controlled trial. Neurologists identified eligible patients, through neurology or MS clinics at the Walton Centre for Neurology and Neurosurgery and satellite clinics. Subjects identified as having a confirmed diagnosis of MS and a relapse requiring admission as either day case or inpatient, for treatment with a three day course of IVMP 1 g/day were included. Block randomisation using blocks of four subjects in a different-subject design was followed. Patients were randomly allocated to treatment or control according to the randomisation list and informed of their study group. Written informed consent was obtained, as was local ethical approval for the study.

Control patients received the equivalent of the current standard ward routine for IVMP management, including three days IVMP, commencing on any weekday. Referral to other disciplines such as general neurological physiotherapy and occupational therapy were addressed within available resources, and patients could be referred for subsequent outpatient therapy if this was deemed appropriate and the service was available in their area.

Treatment patients received a planned, multidisciplinary team assessment, including three days IVMP. All members of the MDT (mainly the MS team members) were aware of this group's admission beforehand, allowing allocation of assessment time. Treatment depended on goals set during the initial assessments. Advice was given for continuing self management after discharge and if appropriate, referral to other agencies on discharge was arranged. Therapy treatment for both groups was not standardised as symptom presentation varied and therapy was patient focused to meet subjects' needs at the time. An array of interventions was offered by the multidisciplinary team, ranging from health promotion advice for people with mild disability to passive stretching exercises taught to carers for subjects with more severe disability, or bladder management techniques for those with continence issues.

\section{Assessments}

All outcome measures were completed on admission, at one month and three months after the first day of IVMP. All assessments were conducted in the physiotherapy department of the study centre. Other data such as demographic information, baseline Expanded Disability Status Scale (EDSS), number of therapy contacts, duration of therapy sessions, and the number of subjects referred on to other agencies on discharge were collected.

The primary outcome measures used were the Guy's Neurological Disability Scale (GNDS), ${ }^{21}$ a questionnaire in which the subject replies yes or no to a series of symptom related questions; and the Amended Motor Club Assessment (AMCA), ${ }^{22}$ which was assessed by the research physiotherapist. At the beginning of the study it was decided by review of the available literature not to use the upper limb section of the AMCA as it had been shown to have a ceiling effect in an MS population. ${ }^{22}$ The secondary measures were all self report and comprised the Barthel Index (BI), ${ }^{23}$ the Human Activity Profile (HAP), which comprises a maximum score and adjusted score domain, ${ }^{24}$ and the Short Form 36 Item Health Survey (SF$36)^{25}$ (see table 1). Because of the nature of the study, neither the patients nor the assessing therapist could be blinded; however, only the AMCA was not self reported by the patient.

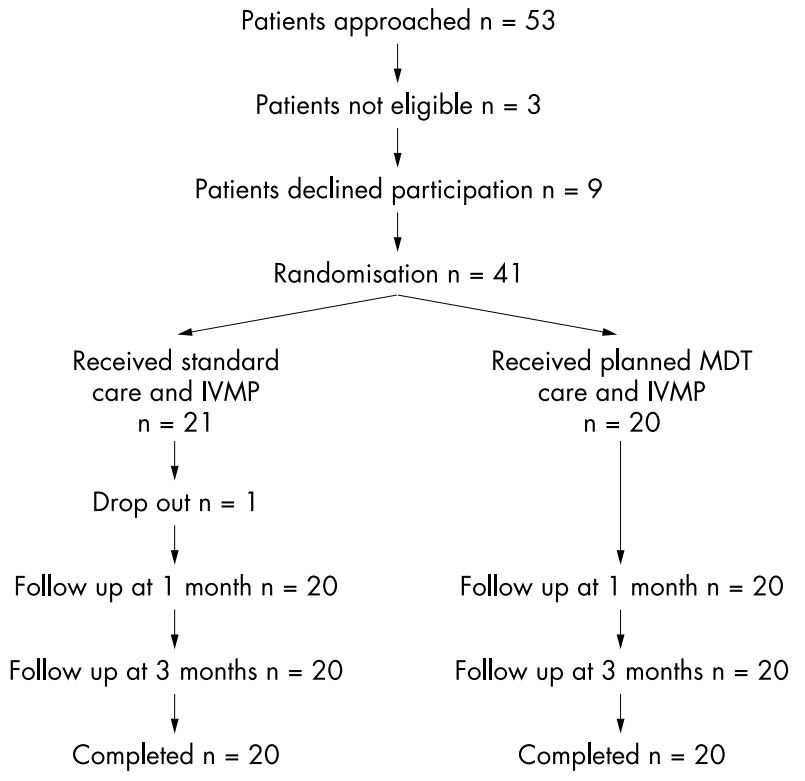

Figure 1 Participant flowchart. 
Table 2 Baseline results-treatment versus control

\begin{tabular}{|c|c|c|c|c|}
\hline & Treatment group $(n=20)$ & & Control group $(n=20)$ & \\
\hline \multicolumn{5}{|l|}{ Gender } \\
\hline Female & 11 & & 16 & \\
\hline Male & 9 & & 4 & \\
\hline Mean age at entry & 38 years $(26-59)$ & $\mathrm{SD}=8.72$ & 42 years $(22-67)$ & $S D=11.09$ \\
\hline Mean time since diagnosis & 7.42 years $(0.33-24.11)$ & $\mathrm{SD}=6.94$ & 5.69 years $(0-19.02)$ & $S D=5.09$ \\
\hline \multicolumn{5}{|l|}{ Type of admission } \\
\hline Inpatient & 11 & & 12 & \\
\hline Outpatient & 9 & & 8 & \\
\hline Mean no. of days since onset of relapse & 45.95 days $(6-48)$ & $S D=21.97$ & 38.35 days (10-105) & $S D=26.92$ \\
\hline Mean baseline EDSS & $5.4(2-7.5)$ & $S D=1.87$ & $5.1(2.5-8.0)$ & $S D=1.86$ \\
\hline \multicolumn{5}{|l|}{ EDSS group } \\
\hline Mild = 0-3.5 & Mild $=6$ & & Mild $=5$ & \\
\hline Moderate $=4.0-6.5$ & Moderate $=10$ & & Moderate $=11$ & \\
\hline Severe $=7.0-10.0$ & Severe $=4$ & & Severe $=4$ & \\
\hline
\end{tabular}

Due to staff mix within the service, the assessing therapist was also the treating therapist. Levels of accepted clinical significance for each measure were established via a pilot study $^{26}$ and analysis of previously published data ${ }^{27}$; table 1 indicates this level where available.

\section{Statistical methods}

All data was entered onto a database on SPSS version 9.0 for Windows. It was established, with statistical advice, that any study effect would be shown at three months compared to baseline; therefore analysis was conducted on this change score data. Initial statistical analysis involved a SchapiroWilks test for normality of data. Only the GNDS data were of normal distribution and were tested parametrically (univariate analysis of variance). The AMCA and the secondary measures data were tested non-parametrically (Mann-Whitney test). Other tests completed included establishing effect size of the GNDS and clinical significance of each measure.

\section{RESULTS}

Between September 1999 and February 2001, 53 patients were referred for the study. Of these, three were not eligible, as they were not in a definite relapse. Nine further subjects declined participation for various reasons, including the distance to travel for the assessments and prior commitments. In total 41 patients were recruited to the study. Following baseline measurement one subject dropped out of the study for personal reasons. Forty subjects (20 treatment, 20 control) therefore completed data collection to three months and this data is reported (see fig 1 ).
Analysis revealed that the cohort was representative of the relapsing MS population in terms of gender, time since diagnosis, and disability level. Twenty seven females were recruited. Mean time since diagnosis was 6.4 years (range 0-24.1 years). Mean EDSS at the time of presenting with relapse was 5.25 (range 2-8). Further analysis established that there were no significant differences between the two groups. Table 2 describes the groups at baseline.

Analyses of baseline mean score data for the primary measures revealed that the groups had no statistically significant differences in level of disability. Table 3 shows the mean score for the primary measures over the study period.

The difference in mean change score from baseline to three months between treatment and control groups was established and statistically tested with a 95\% confidence interval. The GNDS and AMCA both showed a significance of $p<0.05$. Table 4 shows the results. Effect size was established for the GNDS; it was -1.12 for the treatment group and -0.24 for the control group.

Mean scores for both groups in the $\mathrm{BI}, \mathrm{HAP}$, and four domains of the SF-36 (Physical Function (PF), Social Function (SF), Role Emotional (RE), and Mental Health $(\mathrm{MH})$ ) followed a similar sustained improvement trend as those of the primary measures (see table 3 ). The other four domains (Role Physical (RP), Energy (E), Pain (P), and General Health $(\mathrm{GH})$ ) differed in that these mirrored those of the control group, presenting an improvement at one month but a decline at three months (see table 3 ).

Analysis of change score data for the secondary measures indicates significant differences of $p<0.05$ in the HAP (both

Table 3 Mean scores (SD) for all measures

\begin{tabular}{|c|c|c|c|c|c|c|}
\hline \multirow[b]{2}{*}{ Measure } & \multicolumn{3}{|l|}{ Treatment } & \multicolumn{3}{|l|}{ Control } \\
\hline & Baseline & 1 month & 3 months & Baseline & 1 month & 3 months \\
\hline GNDS & $21.1(7.1)$ & $14.1(6.9)$ & $13.1(8.9)$ & $21.5(7.2)$ & $18.5(8.2)$ & $19.7(10.6)$ \\
\hline AMCA & $56.1(15.4)$ & 67.5 (10.3) & 69.1 (6.9) & $48.0(13.8)$ & 57.6 (12.7) & 54.3 (18.0) \\
\hline HAPM & $54.4(20.2)$ & $67.9(13.4)$ & 69.9 (14.2) & $58.3(16.2)$ & $61.6(17.8)$ & $54.5(25.1)$ \\
\hline HAPA & 35.1 (19.0) & $48.1(20.2)$ & $53.2(20.7)$ & $33.2(15.4)$ & $40.6(20.6)$ & $36.7(23.7)$ \\
\hline $\begin{array}{l}\text { BI } \\
\text { SF-36 }\end{array}$ & $14.8(2.7)$ & $17.2(2.3)$ & $17.4(2.3)$ & $14.7(2.2)$ & $15.8(2.6)$ & $15.1(4.1)$ \\
\hline PF & 23.5 (19.2) & $38.7(24.4)$ & $45.5(29.1)$ & 23.7 (17.2) & 36.7 (29.9) & $33.0(29.2)$ \\
\hline SF & $34.3(26.0)$ & $63.8(21.8)$ & $68.4(25.6)$ & $34.8(21.2)$ & $62.2(27.0)$ & 50.1 (33.5) \\
\hline $\mathrm{RP}$ & $5.0(13.1)$ & $38.7(45.6)$ & $35.0(38.4)$ & $11.2(28.6)$ & $20.0(33.1)$ & $27.5(42.1)$ \\
\hline RE & 33.3 (45.9) & $66.6(44.6)$ & $66.6(44.6)$ & $45.0(47.5)$ & $65.0(41.2)$ & $55.0(48.7)$ \\
\hline $\mathrm{MH}$ & $56.2(16.3)$ & $70.8(21.2)$ & $75.6(17.6)$ & $58.6(20.6)$ & $69.2(18.4)$ & $64.3(25.0)$ \\
\hline$E$ & 29.2 (17.0) & $51.4(20.9)$ & $48.2(22.4)$ & 25.0 (19.9) & $41.7(20.5)$ & 34.0 (24.7) \\
\hline$P$ & $67.1(25.6)$ & $85.1(15.4)$ & $78.4(24.9)$ & $43.3(25.4)$ & $64.3(25.1)$ & 50.5 (27.5) \\
\hline $\mathrm{GH}$ & 43.8 (21.9) & $55.6(25.3)$ & $52.8(24.3)$ & $45.6(23.5)$ & $51.2(23.9)$ & 44.2 (29.8) \\
\hline
\end{tabular}

SF-36 domains: PF, Physical Function; SF, Social Function; RP, Role Physical; RE, Role Emotional; MH, Menta Health; E, Energy; P, Pain; GH, General Health. 


\begin{tabular}{|c|c|c|c|c|c|c|c|}
\hline Measure & Mean & SD & Median & Min & Max & Range & p value \\
\hline \multicolumn{8}{|l|}{ GNDS } \\
\hline Treatment & -8.0 & 8.49 & -8.5 & 13 & -27 & 40 & 0.030 \\
\hline Control & -1.75 & 9.04 & -4.0 & 18 & -17 & 35 & \\
\hline \multicolumn{8}{|l|}{ AMCA } \\
\hline Treatment & 13.0 & 10.91 & 8.0 & 1 & 40 & 39 & 0.035 \\
\hline Control & 6.40 & 13.28 & 3.5 & -11 & 41 & 52 & \\
\hline \multicolumn{8}{|l|}{ HAPM } \\
\hline Treatment & 15.3 & 18.07 & 14.50 & 0 & 66 & 66 & 0.004 \\
\hline Control & -3.85 & 19.46 & 0.00 & -55 & 24 & 79 & \\
\hline \multicolumn{8}{|l|}{ HAPA } \\
\hline Treatment & 18.15 & 19.85 & 14.50 & -4 & 62 & 66 & 0.019 \\
\hline Control & 3.45 & 15.12 & 2.00 & -24 & 34 & 58 & \\
\hline \multicolumn{8}{|l|}{$\mathrm{BI}$} \\
\hline Treatment & 2.55 & 2.13 & 2.00 & -1 & 7 & 8 & 0.018 \\
\hline Control & 0.40 & 3.11 & 0.50 & -10 & 4 & 14 & \\
\hline
\end{tabular}

\begin{tabular}{|c|c|c|c|c|c|c|c|}
\hline SF-36 domain & Mean & SD & Median & Min & Max & Range & p value \\
\hline \multicolumn{8}{|l|}{ Physical Function } \\
\hline Treatment & 22.0 & 26.27 & 15.0 & -10 & 75 & 85 & \multirow{2}{*}{0.122} \\
\hline Control & 9.25 & 18.01 & 5.0 & -20 & 45 & 65 & \\
\hline \multicolumn{8}{|l|}{ Social Function } \\
\hline Treatment & 34.1 & 19.93 & 33.0 & -10 & 67 & 77 & \multirow[t]{2}{*}{0.051} \\
\hline Control & 15.2 & 31.94 & 5.5 & -56 & 67 & 123 & \\
\hline \multicolumn{8}{|l|}{ Role Physical } \\
\hline Treatment & 30.0 & 44.86 & 25.0 & -50 & 100 & 150 & \multirow[t]{2}{*}{0.243} \\
\hline Control & 16.25 & 37.41 & 0.00 & -50 & 100 & 150 & \\
\hline \multicolumn{8}{|l|}{ Role Emotional } \\
\hline Treatment & 0.00 & 48.43 & 0.00 & -100 & 100 & 200 & \multirow[t]{2}{*}{0.250} \\
\hline Control & -10.0 & 39.12 & 0.00 & -100 & 67 & 167 & \\
\hline \multicolumn{8}{|l|}{ Mental Health } \\
\hline Treatment & 19.35 & 22.17 & 18.0 & -12 & 60 & 72 & \multirow{2}{*}{0.155} \\
\hline Control & 5.7 & 26.80 & 8.0 & -56 & 44 & 100 & \\
\hline \multicolumn{8}{|l|}{ Energy } \\
\hline Treatment & 19.00 & 24.47 & 15.00 & -25 & 55 & 80 & \multirow{2}{*}{0.217} \\
\hline Control & 9.00 & 27.89 & 7.5 & -40 & 65 & 105 & \\
\hline \multicolumn{8}{|l|}{ Pain } \\
\hline Treatment & 11.3 & 27.94 & 5.5 & -56 & 56 & 112 & \multirow[t]{2}{*}{0.494} \\
\hline Control & 7.15 & 19.29 & 5.5 & -23 & 56 & 79 & \\
\hline \multicolumn{8}{|l|}{ General Health } \\
\hline Treatment & 8.95 & 16.2 & 7.5 & -32 & 37 & 69 & \multirow[t]{2}{*}{0.159} \\
\hline Control & -1.4 & 26.21 & 0.00 & -72 & 47 & 119 & \\
\hline
\end{tabular}

maximum score and adjusted score) and BI (see table 4). The SF-36 domains did not show any significance, although the Social Function domain did indicated a trend towards significance $(p=0.051)($ see table 5$)$.

\section{Therapy intervention}

The duration of therapy and specialist nursing intervention given to the groups differed according to the group protocols, as did the number of subjects referred to local services following steroid treatment. Referrals to other disciplines within the study centre also varied between groups. Table 6 summarises the differences between the groups.

Physiotherapy was the most common intervention administered following initial assessment and had the longest duration of treatment among those given. The physiotherapy intervention offered included provision of mobility aids; exercise programmes for stability, posture, balance, and fitness; health education; and assessment for provision of orthotics. Occupational therapy input included provision of equipment for the home; fatigue and stress management; provision of small aids; and referral to social services for more long term home assessments. Bladder management and advice on coping mechanisms were the main interventions offered by the specialist nurses.

\section{DISCUSSION}

This unique randomised controlled trial aimed to investigate the relative benefits of patients with MS relapse receiving a short course of focused, MDT care in combination with IVMP in comparison to standard care and IVMP. It was conducted to address a gap in knowledge identified in clinical practice and in current literature on MS management. ${ }^{20}$

Of the subjects screened, $80 \%$ entered the study and $97 \%$ of these completed the study. The sample included subjects treated both as inpatients and day case patients in order to reflect standard practice. This research attempted to establish whether access to enhanced therapy in combination with IVMP produced a preferable outcome. It was therefore important to ensure that standard care for the control group did not change from standard care prior to the trial commencing. Data on standard care were established during the pilot study. ${ }^{26}$ The referral rate, duration of treatment, and length of stay recorded for the control group indicated that standard care did not alter during the study period. 
Table 6 Therapy data

\begin{tabular}{lll}
\hline & Treatment group & Control group \\
\hline Mean physiotherapy treatment time; hours (range) & $2.62(1.25-5)$ & $0.26(0-1.30)$ \\
Mean occupational therapy treatment time; hours (range) & $1.49(0.25-4)$ & $0.075(0-0.75)$ \\
No. of subjects seen by speech therapist & $3(15 \%)$ & $0(0 \%)$ \\
No. of subjects seen by MS nurse specialists & $20(100 \%)$ & $9(45 \%)$ \\
No. of subjects seen by orthoptist & $3(15 \%)$ & $0(0 \%)$ \\
No. of subjects referred for further physiotherapy & $13(65 \%)$ & $3(15 \%)$ \\
No. of subjects referred for further occupational therapy & $10(50 \%)$ & $2(10 \%)$ \\
Mean length of stay; days (range) & $3.45(3-8)$ & $4.8(3-14)$ \\
& SD $=1.15$ & SD $=3.07$ \\
\hline & &
\end{tabular}

The distribution of gender, mean age at entry, mean time since diagnosis, and analysis of baseline outcome measure scores indicated no significant differences between the two study groups, suggesting randomisation had been effective.

Some of the improvement in both groups is likely to be spontaneous recovery. A further part of the recovery seen is a direct result of the IVMP itself, by reversing the changes to the blood-brain barrier and minimising symptom presentation and duration. ${ }^{28}$ This accounts for some of the initial improvement that is seen in the control subjects. However, the level of spontaneous and steroid related recovery should be comparable for both groups.

The main distinction accounting for the statistical differences in change score between groups is the therapy intervention. There were significant differences in the overall duration of therapy given to the two groups and distinct differences between groups, in favour of the treatment group, in the number of subjects seen by different disciplines. The therapy input given to the control group reflects current practice on the wards. The higher number of subjects referred on for further treatment in the intervention group is a reflection of the increased contact with therapists during admission. It was difficult within this study to ascertain whether the benefits obtained in the treatment group were accounted for by the acute intervention or the later community management. This study aimed to evaluate the opportunity to access a package of care, and the benefits are suggested to be due to a balance of a combination of acute and later stage rehabilitation reflected in the greater referral to local services in the treated group.

Statistical testing of the change score data from the primary measures showed a correlation between enhanced therapy intervention and an improvement in impairment and disability. The AMCA measures motor impairment. ${ }^{22}$ It is expected that change would be effected through therapy, with specific exercise and retraining of muscle activity. ${ }^{13}$ Changes in range of movement, motor control, and quality of movements become evident. This then has a direct influence on motor functions such as balance and transfers, ${ }^{12}{ }^{13}$ both of which are assessed in the AMCA scoring.

Improvement in motor function at this level is expected to influence disability level. Improved range of movement, balance, and transfer techniques can reduce the dependency on others, promote acquisition of new or adapted skills, enhance independence, and facilitate improved management of other symptom areas through greater control of movement. The GNDS will reflect any changes achieved through PT, OT, MS nurse specialist, or orthoptic intervention.

The BI and HAP showed significant results in favour of the treatment group when mean change score and mean scores at baseline and three months were analysed. The domains of the SF-36 did not show statistical significance. It was anticipated that improving levels of disability, empowerment of the subjects, and increasing participation would reflect on the SF-36 domains. However, as the mean scores for several domains of the SF-36 showed greater improvement in the treatment than in the control group, there is still some benefit from the MDT intervention in altering health status.

Proving statistical significance does not always ensure that the changes will be clinically significant or relevant to the subjects involved. For each measure interpretation of the change score needs to be placed into a clinical context. Clinical significance can be estimated through assessing change score in comparison to a previously set significance level according to earlier data (see table 1 ) or via establishing effect sizes. ${ }^{29}$ Both of these methods were used to establish whether the changes were clinically significant in this patient group. The results highlighted in tables 4 and 6 further support the theory that enhanced therapy improves outcome after steroids by showing, in the primary measures and two of the secondary measures, that changes occurred which were likely to be clinically and functionally relevant to the subjects. The effect size for the GNDS was established. The results showed a large effect size for the treatment group and only a small effect size for the control group,${ }^{28}$ which supports the suggestion that the intervention outcome is clinically relevant in this patient group.

In respect of the SF-36, despite no statistically significant difference, a clinically significant change score in six of the domains in the intervention group (the exceptions being the RE and GH domains) was established. However, only the SF and RP domains represented a clinically significant change score for the control subjects.

The main finding was that steroids and rehabilitation as a planned admission on an inpatient or daycase basis was superior to steroid treatment and standard care alone. There was a statistically significant difference between IVMP with standard care and IVMP with focused MDT care for the two primary measures (AMCA and GNDS) and some of the secondary measures (BI, HAP). This change exceeded predetermined evidence based levels for clinical significance for the GNDS, assessing disability, and the AMCA, assessing motor recovery. The SF-36 showed a trend in favour of the intervention.

The outcome measures were chosen to reflect the changes anticipated from the intervention - that is, alteration in motor function and disability level, and were hypothesis driven. Inability to incorporate assessor blinding into the design was a limitation of the trial. The GNDS score is dictated only by the subject responses and therefore is not influenced by the opinion of the person asking the question. The researcher scores the AMCA according to certain set criteria. Careful adherence to AMCA guidelines minimises but does not abolish intrarater bias. A type I error could potentially have occurred in the AMCA analysis; however, as both the primary measures showed the same trend and the GNDS is self reported by the patient, it is unlikely. The secondary measures that are also all self report concur with this.

The SF-36 especially, but to a lesser degree the BI and AMCA, exhibit floor and ceiling effects. For the AMCA, the range of baseline scores for the study groups indicate a possible ceiling effect still to be present, as many patients were 
scoring in the higher end of the scoring range. The SF-36 has been shown previously $y^{30}$ to exhibit ceiling and floor effects, and the raw data for some subjects concurs with this in certain domains (RP and RE).

Three months was chosen for follow up as it was felt this would allow time for services in the community to be established, equipment required to be put in place, and for subjects to make gradual alterations to their lifestyle as a result of the advice and programme given. It was also felt that three months was soon enough after IVMP treatment that any significant results could be attributed to the intervention and not necessarily to other factors. However, it may be the case that this is still too short a period for any alteration in health related quality of life to be evident.

\section{Conclusions}

In conclusion this randomised controlled trial is the first to show that planned, focused MDT care during steroid treatment of MS relapse is beneficial. Changes in the study design would enhance the results. These include conducting the study as part of a multicentre trial ("standard care" and focused MDT care would need to be more standardised between centres); longer follow up period, such as up to 12 months, to allow potential changes in quality of life to be established and to determine the effects of the intervention over a long period; and use of a second, blinded assessor to establish AMCA score.

The findings suggest that introducing a problem focused team integrated approach to the steroid management of MS relapse in the acute setting, including access to appropriate levels of therapy, is of benefit to patients in terms of motor function, disability, and aspects of health related quality of life.

\section{ACKNOWLEDGEMENTS}

The authors would like to thank all the study participants; the staff at the Walton Centre for Neurology and Neurosurgery, Liverpool for assistance and support in administration of the study; and Serono Pharmaceuticals UK for the grant which supported the research therapists.

\section{Authors' affiliations}

J Craig, C A Young, M Ennis, M Boggild, The Walton Centre for Neurology \& Neurosurgery, Lower Lane, Liverpool, UK

G Baker, University of Liverpool, Liverpool, UK

Competing interests: none declared

\section{REFERENCES}

1 Compston A. Genetic susceptibility to multiple sclerosis. In: Compston A, Ebers $\mathrm{G}$, Lassmann $\mathrm{H}$, et al, eds. McAlpine's multiple sclerosis, 3rd edn. London: Churchill Livingstone, 1998:104-44

2 Lublin FD, Reingold SC. Defining the clinical course of multiple sclerosis: results of an international survey. Neurology 1996;46:907-1 1.

3 Young CA. Improving the care of patients with progressive multiple sclerosis: an evidence-based approach. J R Coll Physicians 2002;32:19-23.
4 Goodkin DE. Role of steroids and immunosuppression and effects of interferon beta-1 b in multiple sclerosis. West J Med 1994;161:292-8.

5 Taggart HM. Multiple sclerosis update. Orthop Nurs 1998:23-9.

6 Barnes D, Hughes RAC, Morris RW, et al. Randomised trial of oral and intravenous methylprednisolone in acute relapses of MS. Lancet 1997:349:902-6.

7 Fillipini G, Brusaferri F, Sibley WA, et al. Corticosteroids or ACTH for acute exacerbations in multiple sclerosis (Cochrane review). The Cochrane Library, Issue 4, Oxford, 2000

8 Tremelett HL, Luscombe DK, Wiles CM. Use of corticosteroids in multiple sclerosis by consultant neurologists in the UK. J Neurol Neurosurg Psychiatry 1998;65:362-5.

9 Stevenson VL, Thompson AJ. The management of MS: current and future therapies. Drugs of Today 1998;34:267-82.

10 Mertin J. Rehabilitation in MS. Ann Neurol 1994;36(suppl):S130-3.

11 De Souza LH, Worthington JA. The effect of long-term physiotherapy on disability in multiple sclerosis patients. In: Rose FC, Jones R, eds. MS immunological, diagnostic and therapeutic aspects. Current problems in neurology, No 3. London and Paris: John Libbey Eurotext 1987:155-67

12 Fuller KJ, Dawson K, Wiles CM. Physiotherapy in chronic MS: a controlled trial. Clin Rehabil 1996;10:195-204.

13 Lord SE, Wade DT, Halligan PW. A comparison of two physiotherapy treatment approaches to improve walking in MS: a pilot randomised control study. Clin Rehabil 1998;12:477-86.

14 Wiles CM, Newcombe RG, Fuller KJ, et al. Controlled randomised crossover trial of the effects of physiotherapy on mobility in chronic MS. J Neurol Neurosurg Psychiatry 2001;70:174-9.

15 Jones L, Lewis Y, Harrison J, et al. The effectiveness of OT and PT in MS patients with ataxia of the upper limb and trunk. Clin Rehabil $1996 ; 10: 277-82$

16 Freeman JA, Langdon DW, Hobart JC, et al. The impact of in-patient rehabilitation on progressive MS. Ann Neurol 1997;42:236-44.

17 Freeman JA, Langdon DW, Hobart JC, et al. Inpatient rehabilitation in multiple sclerosis-do the benefits carry over into the community? Neurology 1999;52:50-6

18 Kidd D, Howard RS, Losseff NA, et al. The benefit of in-patient neurorehabilitation in MS. Clin Rehabil 1995;9:198-203.

19 Kidd D, Thompson AJ. Prospective study of neurorehabilitation in multiple sclerosis. J Neurol Neurosurg Psychiatry 1996;62:423-4.

20 Bethoux F, Miller DM, Kinkel RP. Recovery following acute exacerbations of multiple sclerosis: from impairment to quality of life. Mult Scler 2001;7:137-42.

21 Sharrack B, Hughes RAC. The Guy's Neurological Disability Scale (GNDS): a new disability measure for multiple sclerosis. Mult Scler 1999;5:223-33

22 De Souza LH, Ashburn A. Assessment of motor function in people with MS. Physiother Res Int 1996:1:98-111.

23 Collin C, Wade DT, Davies S, et al. The Barthel ADL Index: a reliability study. Int Disabil Stud 1988;10:61-3.

24 Daughton D, Fix AJ, Kass I, et al. Maximum oxygen consumption and the ADAPT quality of life scale. Arch Phys Med Rehabil 1982;63:620-2.

25 Ware JE, Sherbourne CD. The MOS 36-item short-form health survey (SF-36): I conceptual framework. Med Care 1992;30:473-83.

26 Craig J, Young CA, Boggild $M$, et al. A study comparing multidisciplinary rehabilitation against standard therapy in multiple sclerosis patients receiving steroid treatment after relapse. Mult Scler 1999;5:S129.

27 Hoogervorst EJL, Kalkers NF, Van Winsen LML, et al. Differential treatment effect on measures of neurologic exam, functional impairment and patient self report in multiple sclerosis. Mult Scler 2001;7:335-9.

28 Miller CM, Hens M. MS: a literature review. J Neurosci Nurs 1993;25:174-9.

29 Kazis LE, Anderson JJ, Meenan RF. Effect sizes for interpreting changes in health status. Med Care 1989;27(suppl): 178-89.

30 Freeman JA, Langdon DW, Hobart JC, et al. Health related quality of life in people with MS undergoing inpatient rehabilitation. J Neurol Rehabil 1996;10:185-94.

31 Freeman JA, Hobart JC, Langdon DW, et al. Clinical appropriateness: a key factor in outcome measure selection: the 36 item short form health survey in multiple sclerosis. J Neurol Neurosurg Psychiatry 2000;68:150-6. 